\title{
Nonlocal Analysis of Natural Vibrations of Carbon Nanotubes
}

\author{
Małgorzata Chwał \\ (Submitted November 22, 2017; in revised form September 1, 2018; published online October 1, 2018)
}

\begin{abstract}
When the atomic level is considered, the dynamic properties have a major influence on nanostructures behavior because of their ultrahigh or very high natural frequencies. Vibrations are essential in the analysis of resonators, oscillators, and sensors for carbon nanotube-based devices. The present paper is dedicated to the eigenfrequencies analysis of single-walled carbon nanotubes. In the analysis, the small-scale coefficient is introduced, and the nonlocal elasticity theory is applied in the modal analysis of the carbon nanotubes. The correlation between nonlocal small-scale parameter and the vibrational behavior of the carbon nanotubes is studied. The determination of nonlocal parameter is based on the structural finite element model of the nanotube. A detailed parametric study is realized to investigate the effects of the length-to-diameter ratio $L /$ $D$, nonlocal coefficient, the influence of boundary conditions on eigenfrequencies of carbon nanotubes. The main contribution of this work is the evaluation of the nonlocal parameter for single-walled carbon nanotubes based on the finite element approximation in modeling the dynamic behavior instead of commonly used MD simulations. It was clearly displayed that the application of the nonlocal beam models involves analysis of the small-scale parameter, whose value determines the final eigenfrequencies. The highest values of eigenfrequencies were observed for the local beam model. It was presented that the eigenfrequencies for carbon nanotubes decrease when the nanotube length increases for all analyzed modes. On the other hand, when the diameter of carbon nanotubes increases, the eigenfrequency values rise. The numerical validation of the nonlocal parameter revealed the high dependence on the boundary conditions and the mode number. However, the influence of the mode number and the boundary conditions is vanishing for long single-walled carbon nanotubes.
\end{abstract}

Keywords computational materials development, dynamic, materials by design, mechanical, modeling and simulation, nanomaterials

\section{Introduction}

Firstly described by Iijima and Iijima and Ichihashi (Ref 1, 2) carbon nanotubes (CNTs) are presented as perfect systems for exploring electromechanical effects because of a small diameter, low mass, and defect-free structure on the molecular level (Ref 3). Moreover, some experimental reports on CNTs revealed high values of natural frequency (Ref 4, 5). The outstanding properties of CNTs allow their use as highfrequency nanoelectromechanical systems (NEMS) (Ref 3, 6$8)$. For some NEMS, the oscillation frequency is a key property (Ref 6). Also, the values of natural frequencies or shape modes can be used as an indirect method of Young's modulus evaluation (Ref 7). Considering the carbon nanotube-based nanoelectromechanical systems, their vibrations are essential in

This article is an invited paper selected from presentations at "11th International Conference on Advanced Computational Engineering and Experimenting, ACE-X 2017," held July 3-6, 2017, in Vienna, Austria, and has been expanded from the original presentation.

Małgorzata Chwal, Institute of Machine Design, Cracow University of Technology, Warszawska 24, 31-155 Cracow, Poland. Contact e-mail: malgorzata.chwal@pk.edu.pl. the analysis, for instance, field emission devices, sensors, resonators, oscillators, charge detectors, and also biosensors (Ref 8). During carbon nanotube deformation, the nanotube diameter and length are changed which causes the resonant frequency modifications (Ref 9). Also, the shift of the resonant frequency of a nanotube resonator is dependent on the force the nanotube is subjected. Thus, the most crucial issue is first of all to identify the resonant frequency of nanotube and next to formulate the correlation between resonant frequency shift and the applied force. In biosensor with carbon nanotubes, the natural vibrations and mode shapes are an excellent measure to describe the dynamic behavior of the cantilevered or bridged carbon nanotubes under biochemical interactions (Ref 10-12).

In the theoretical studies on CNT vibrations, the following models might be illustrated: (1) atomic, (2) continuum, and (3) mix atomic/continuum models. From the engineering point of view, the atomic models are not sufficient because of the scale and time limitation and in fact, these models are applied only in the case of short CNTs investigations - see Ansari et al. (Ref 13). When the carbon nanotube continuum modeling is reviewed, beam and shell models might be found, for example, Yoon et al. (Ref 14) and Wang et al. (Ref 15). Typically, the flexural eigenfrequencies and mode shapes are obtained using beam models, whereas shell models are applied for the radial, axial, and circumferential eigenfrequencies and modes extractions (Ref 8). Also, atomic-structural approaches based on the molecular dynamics and continuum mechanics are developed, for example, $\mathrm{Li}$ and Chou (Ref 16). In contrast to the continuum models, not only geometry of carbon nanotubes but also interatomic interactions are taken into account in the atomic-structural models. Characteristic is that the eigenfre- 
quencies of nanotubes are on the level of terahertz values and it is strongly depended on the applied boundary conditions (BC) (Ref 8). However, Ambrosini and Borbon (Ref 17) presented beam model based on the theory for the thin-walled beam that considers shear effects and is independent on the boundary conditions.

Based on an equivalent continuum mechanics approach, Lee et al. (Ref 18) evaluated the fundamental natural frequency of single-walled carbon nanotubes (SWCNTs). They reported the frequencies for armchair nanotubes varied between 7 and $300 \mathrm{GHz}, 40$ and $1700 \mathrm{GHz}$, and 20 and $800 \mathrm{GHz}$ for SWCNTs under clamped-free (C-F), clampedclamped $(\mathrm{C}-\mathrm{C})$, and simply supported $(\mathrm{S}-\mathrm{S})$ boundary conditions, respectively. A molecular dynamics study was employed by Ansari et al. (Ref 13) to investigate the vibrations of single- and double-walled carbon nanotubes (DWCNTs). They observed that the natural frequency and its dependence on boundary conditions are reduced by increasing the tube length. It was revealed that the natural frequency of DWCNTs lies between those of the constituent inner and outer SWCNTs and it is close to those of the outer one. The presented values varied from 31 to $247 \mathrm{GHz}$ for long and short SWCNTs with $\mathrm{C}-\mathrm{F}$ boundary conditions. Whereas for $\mathrm{C}-\mathrm{C}$ boundary conditions, the natural frequencies were higher and varied from 118 to $1167 \mathrm{GHz}$ for long and short SWCNTs. A structural mechanics approach was applied by Sakhaee-Pour et al. (Ref 19) and Parvaneh et al. (Ref 20) to investigate the vibrational stability of SWCNTs. Finite element method (FEM) studies on the natural frequencies of single- and multi-walled carbon nanotubes (MWCNTs) using Euler-Bernoulli beam elements were performed, e.g., by Fan et al. (Ref 21), Rahmandoust and Öchsner (Ref 22) and Ghavamian and Öchsner (Ref 23). Fan et al. (Ref 21) presented the numerical examples for approximating the mechanical properties of nanotubes including Young's moduli, shear modulus, natural frequency, buckling loads for MWCNT. Rahmandoust and Öchsner (Ref 22) studied buckling behavior and resonant frequency modes of the armchair and zigzag models of SWCNT. Later, in work (Ref 23), it was revealed that by increasing the number of carbon nanotube walls, their natural frequencies raise which means that the higher the number of walls is, the more stable the structure becomes against vibration. It was also showed that armchair CNTs have higher natural frequencies than zigzag structures.

Concerning CNTs, it is observed that classical (local) beam theories are insufficient for the mechanical properties analysis of small beam-like structures in the absence of any material length scale parameters, for example, Peddieson et al. (Ref 24), Anderson et al. (Ref 25), Reddy (Ref 26), Wang et al. (Ref 27), Heireche et al. (Ref 28) and Civalek and Demir (Ref 29). Size effects often become prominent at nanometer scales. The problem lies in the fact that classical beam models cannot capture the small-scale effect in the mechanical properties presenting scale-free relations. Therefore, some non-classical continuum approaches have been defined to determine the mechanical behavior of small-sized structures, such as nonlocal elasticity theory (Ref 24, 26-35), strain gradient theories (Ref 36-41), and couple stress theory (Ref 42, 43).

Applications of nonlocal continuum mechanics for CNTs have been studied by many researchers in the issue of static (Ref 27, 28) and dynamic analyses (Ref 25, 30). Wang (Ref 44) pointed out that nonlocal elasticity theory should be used for an accurate prediction of wave propagation in CNTs. Wang and Varadan (Ref 45) analyzed vibration of both SWCNTs and DWCNTs via nonlocal Euler-Bernoulli (E-B) and Timoshenko (TB) beam models. Lu et al. (Ref 46) and Heireche et al. (Ref 47) studied wave propagation in nanotubes used in nonlocal elasticity beam models. Arash and Wang (Ref 48) discussed the superiority of nonlocal continuum models to their local counterparts, the necessity of the adjustment of the small-scale parameter, and the applicability of nonlocal continuum models. Barretta and Sciarra (Ref 49) presented the nonlocal elastostatic problem of Euler-Bernoulli nanobeams and pointed out the nonlocality effect. Hu et al. (Ref 50) used nonlocal shell model to study wave propagation in CNTs and compared it with MD results. Fazelzadeh and Ghavanloo (Ref 51) applied nonlocal anisotropic elastic shell model for vibrations of SWCNTs. The nonlocal FEM analysis of Euler-Bernoulli and Timoshenko nonlocal beam was performed by Pradhan (Ref 52). A review paper on the nonlocal elasticity focusing on main results and future challenges was published by Di Paola et al. (Ref 53). Recently, Eltaher et al. (Ref 54) published also a review on nonlocal elastic models for bending, buckling, vibrations, and wave propagation of nanoscale beams. In general, nonlocal descriptions led to more flexible mechanical behavior of nanostructures than the local form except cases when paradoxes appear-see, for example, Fernandez-Saez et al. (Ref 55), Koutsoumaris et al. (Ref 56) and Romano et al. (Ref 57).

In the current work, the analytical calculations of eigenfrequencies of carbon nanotubes based on the local and nonlocal Euler-Bernoulli beam theory were presented. Additionally, a FEM analysis of eigenfrequency was conducted. The motivation for the present study is to propose an evaluation of nonlocal parameter through fitting the analytical beam model results to FEM predictions involving a space frame model of SWCNTs. The main contribution of this work is the computation of the nonlocal parameter for SWCNTs based on the comparison between the FEM results and nonlocal analytical predictions for natural vibrations. The evaluation of nonlocal parameter for carbon nanotubes presented in the literature is mainly based on the comparison of analytical predictions with MD modeling. MD simulations are computationally expensive. On the other hand, experimental studies are cumbersome and difficult to conduct at the nano-level. Thus, in the present paper, the nonlocal parameter is verified by the FEM studies of natural vibrations for CNT instead of MD analysis. Also, parametric calculations are realized to investigate the influence of the length-to-diameter ratio $L / D$, nonlocal parameter value, the influence of boundary conditions on natural frequencies of SWCNTs. The strong influence of boundary conditions and mode numbers on the proposed nonlocal parameter is presented and discussed.

\section{Nonlocal Continuum Mechanics}

Eringen (Ref 58) and Eringen and Edelen (Ref 59) first introduced the nonlocal elastic continuum mechanics defining that the stress state at a given point $r$ depends not only on the local stress at that point but also on the function of the stress state at all points in the body. For homogenous and isotropic elastic solids with zero body forces, the fundamental nonlocal equations are written as (Ref 45): 
$\sigma_{i j, j}=0$

$\sigma_{i j}(r)=\int \alpha\left(\left|r^{\prime}-r\right|, \tau\right) \sigma_{i j}^{\prime}\left(r^{\prime}\right) d V\left(r^{\prime}\right)$

$\varepsilon_{i j}=\frac{1}{2}\left(u_{i, j}+u_{j, i}\right) \quad i, j=1,2,3$

where $\sigma_{i j}(r)$ nonlocal stress tensor at point $r, \sigma_{i j}^{\prime}\left(r^{\prime}\right)$ is local (classical) stress at any particular points $r$ ' within domain $V$, whereas $\alpha\left(\left|r^{\prime}-r\right|, \tau\right)$ is the nonlocal modulus that depends on the distance between $r$ and $r$ and dimensionless length scale $\tau$ expressed as $\tau=e_{0} a / L$. The $e_{0}$ is a nonlocal material parameter appropriate to each material, $a$ and $L$ are the internal and external characteristic lengths, respectively. Mostly, in the case of carbon nanotubes, $a=1.42 \mathrm{~nm}$ is the carbon-carbon bond length, and $L$ is described as the length of the carbon nanotube.

As an alternative approach instead of the nonlocal integral Eq 1, an equivalent second-order differential constitutive relation is described by $(\operatorname{Ref} 30)$ :

$\sigma-\left(e_{0} a\right)^{2} \nabla^{2} \sigma=\sigma^{\prime}$

where $\nabla$ is the Laplace operator. The critical issue in the nonlocal studies is to estimate the magnitude of the nonlocal material parameter $e_{0}$. The magnitude of the nonlocal material parameter is determined experimentally or estimated by matching the dispersion curves of plane waves with those of atomic lattice dynamics (Ref 60).

\section{Fundamental Relations for Local and Nonlocal Beam Theory}

The local (classical) Euler-Bernoulli beam equation of motion of free vibration of carbon nanotubes is given by:

$\rho A \frac{\partial^{2} w}{\partial t^{2}}+E I \frac{\partial^{4} w}{\partial x^{4}}=0$

where $t$ and $x$ are time and the axial coordinate, respectively; $w$ is the deflection of SWCNT; $A$ is the cross-sectional area and $\rho$ is the mass density of SWCNT; $E$ and $I$ are the elastic modulus and the moment of inertia of a cross section, respectively. The displacements of the vibrational solution in the SWCNT are described by:

$w=X(x) e^{i \omega t}$

where $\omega$ is the circular frequency. Substituting Eq 4 into Eq 3, we obtain an equation of vibrational properties of SWCNT:

$X(x)=A \sin (k x)+B \cos (k x)+C \sinh (k x)+D \cosh (k x)$

where $A, B, C$, and $D$ are constants. The coupling relation is as follows:

$\omega^{2}=k^{4} \frac{E I}{\rho A}$

in which $k$ is a frequency parameter. The solution of Eq 5 depends on boundary conditions (BC) affected by support of a beam. Solving Eq 5 for different boundary conditions, the relations for eigenfunctions, eigenvalues, and eigenfrequencies can be obtained. The applied boundary conditions (Ref 60) for nanobeam with the length $L$ are presented in Table 1 .

Reddy (Ref 26) reformulated the equation of motion of various kinds of beam theories available (Euler-Bernoulli, Timoshenko, Reddy, and Levinson) using the nonlocal differential constitutive relations of Eringen. The nonlocal EulerBernoulli beam equation of motion of a free vibration in carbon nanotubes is given by ( Ref 50$)$ :

$\rho A \frac{\partial^{2}}{\partial t^{2}}\left(w-\left(e_{0} a\right)^{2} \frac{\partial^{2} w}{\partial x^{2}}\right)+E I \frac{\partial^{4} w}{\partial x^{4}}=0$

The local (classical) Euler-Bernoulli beam equation is obtained when the relation $e_{0} a=0$ in Eq 7. For example, the circular vibrations for the simply supported nonlocal beam may be presented in the following way ( $\operatorname{Ref} 41)$ :

$\omega=\sqrt{\frac{1}{\lambda}} \frac{n^{2} \pi^{2}}{L^{2}} \sqrt{\frac{E I}{\rho A}}, \lambda=1+\left(e_{0} a\right)^{2} \frac{n^{2} \pi^{2}}{L^{2}}$

The angular frequency $\omega$ is computed according to Eq 8, and frequency $f$ using relation $f=\omega / 2 \pi$.

The value of the nonlocal parameter has the crucial influence on the final results. As far as we know, there are no experimental studies to calculate the magnitude of $e_{0}$ for CNTs. There are some suggestions in the literature how to define the nonlocal scale parameter $(\operatorname{Ref} 60)$. Instead of determining the $e_{0}$ precisely, there are some works in which the $e_{0} a$ value is evaluated based on the frequency value obtain from the theoretical models or based on the available experimental frequencies. Eringen (Ref 61) associated the frequency given by Born-Karman model of lattice dynamics and that of nonlocal theory for plane waves and obtained a value of 0.39 for $e_{0}$. Wang (Ref 44) calculated the scale parameter $e_{0} a$ lower than $2.1 \mathrm{~nm}$ based on the Yoon et al. (Ref 14) experimental observations where the measured frequency value for CNT was about 10 THz. However, based on the Krishnan et al. (Ref 62) experiment, the frequencies for CNTs are about $0.1 \mathrm{THz}$, and the scale parameter $e_{0} a$ is lower than $210 \mathrm{~nm}(\operatorname{Ref} 60)$. It is visible that the $e_{0} a$ value belongs to the wide range what is explained by the different vibration frequencies for various CNTs. In the literature, the suggested scale coefficient values are $0 \leq e_{0} a / L<0.8(\operatorname{Ref} 60)$, and $e_{0}=10.5$ for $L<3.5 \mathrm{~nm}$ (Ref 50) to match Euler-Bernoulli beam results to the MD simulations. The nonlocal effect is particularly visible for short carbon nanotubes (Ref 50).

Table 1 Boundary conditions applied to nanobeam having length $L$

\begin{tabular}{lc} 
Nanobeam support & Boundary condition \\
\hline Simply supported (S-S) & $X(0)=X(L)=0$ \\
& $X^{\prime \prime}(0)=X^{\prime \prime}(L)=0$ \\
Clamped-clamped (C-C) & $X(0)=X(L)=0$ \\
& $X^{\prime}(0)=X(L)=0$ \\
Cantilevered (C-F) & $X(0)=X^{\prime}(0)=0$ \\
& $X^{\prime \prime}(L)=X^{\prime \prime}(L)=0$
\end{tabular}




\section{Eigenfrequencies Versus Nonlocal Scale Parameter}

To analyze the eigenfrequencies of SWCNTs, the analytical and numerical studies were conducted. In the analytical computations, the local and nonlocal Euler-Bernoulli beam model were used, and the results for various nonlocal scale parameters were presented. Because of the small diameter of SWCNT compared to tube wall thickness, the circular continuum beam model was applied, as shown in Fig. 1. In next chapter, the analytical results will be compared with values from the numerical FEM model of the dynamic behavior of CNT as to find the most suitable description of CNT and investigate the influence of small-scale parameter on the eigenfrequencies. To present the comparative studies, firstly the classical vibrational beam analysis is put forward. In the analytical computations, for the short circular beam models of $(5,5)$ and $(10,10)$ SWCNTs, the material and geometrical data presented in Table 2 are used.

In the present research on the small-scale effect on the vibrational behavior of CNT, the applied nonlocal parameter $e_{0}$ was in the range $0 \leq e_{0} \leq 10$ imposing the dimensionless length scale parameter in the range $0 \leq e_{0} a / L \leq 0.5$ for $a=0.142 \mathrm{~nm}$ and $L=2.83 \mathrm{~nm}$ (ratio a/L=0.05). The analyzed range of $e_{0} a / L$ is close to the values proposed by Wang et al. (Ref 60) for high frequencies of CNT observed experimentally.

To consider the influence of small-scale parameter on the dynamic behavior of CNT, the local and nonlocal EulerBernoulli beam model (see Fig. 1) was studied for simply supported (S-S), bridged (C-C) and cantilevered (C-F) boundary conditions - see Table 1 . Some representative results of eigenfrequencies for the local and nonlocal Euler-Bernoulli beam model for various $e_{0} a / L$ and S-S boundary conditions are presented in Fig. 2.

When the nonlocal material parameter is introduced, a lowering of eigenfrequencies is observed. The highest values are for the local beam model. Comparing the local and the nonlocal Euler-Bernoulli vibrational models, it is visible that the small-scale parameter makes the structure more flexible. The variations of eigenfrequency ratio versus nonlocal parameter for various boundary conditions for $(5,5)$ CNTs using material data from Table 2 are presented in Fig. 3. The eigenfrequency ratio was computed as:

Eigen frequency ratio $=\frac{\text { nonlocal eigenfrequency }}{\text { local eigen frequency }}$

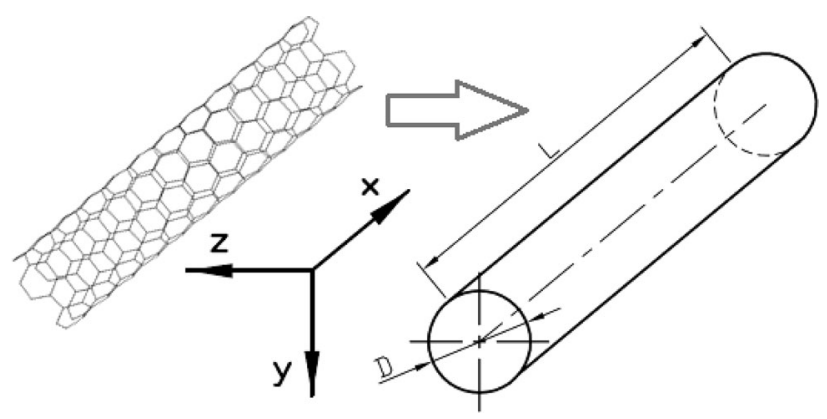

Fig. 1 Continuum modeling of SWCNT: lattice structural model (left) and circular continuum Euler-Bernoulli nanobeam model (right)
It is observed that the increase in the small-scale parameter $e_{0}$ results in the decrease in eigenfrequencies. The decrease varies and depends on the boundary conditions and mode number. The eigenfrequencies for higher modes are more sensitive to the value of $e_{0}$ parameter. Finally, a faster decrease in the free vibration values is visible for higher modes. For the cantilevered nanotube, the lowest deterioration of eigenfrequencies versus $e_{0}$ for the first mode is observed.

The influence of carbon nanotube length and diameter on eigenfrequencies was also studied. The analysis considered two cases of diameter $D=0.678 \mathrm{~nm} \quad((5,5) \quad$ SWCNT $)$ and $D=1.356 \mathrm{~nm}((10,10)$ SWCNT $)$ and nanotubes having a length from range $2.83 \mathrm{~nm} \leq L \leq 28.3 \mathrm{~nm}$-see Fig. 4. It can be seen that the eigenfrequencies for carbon nanotubes decrease when the nanotube length increases for all analyzed modes.

On the other hand, when the diameter of SWCNTs increases also the eigenfrequency values rise. The influence of mode number on eigenfrequencies is mostly visible for short nanotubes for both $(5,5)$ and $(10,10)$ case.

The dimensionless resonant eigenfrequencies in function of nanotube length for various nonlocal parameters from the analyzed range $0 \leq e_{0} \leq 10$ are presented in Fig. 5 for S-S nanobeam case. We can observe the higher the nonlocal parameter is, the lower the eigenfrequency is. Moreover, as the nanotube length increases, the influence of nonlocal parameter on dimensionless natural frequencies becomes less visible. To present some representative results underlining the influence of boundary conditions, the variation of dimensionless resonant eigenfrequencies versus nanotube length is presented in Fig. 6 for $(5,5)$ nanobeam assuming nonlocal parameter $e_{0}=2$. For

Table 2 Material and geometric data of $(5,5)$ and $(10,10)$ SWCNTs

\begin{tabular}{ll}
\hline Parameter & \multicolumn{1}{c}{ Value } \\
\hline Young's modulus $E$ & $1 \times 10^{9} \mathrm{~N} / \mathrm{m}^{2}$ \\
Poisson's ratio $v$ & 0.3 \\
Density $\rho$ & $1200 \mathrm{~kg} / \mathrm{m}^{3}$ \\
Diameter $D$ & $0.674 \times 10^{-9} \mathrm{~m}(5,5)$ \\
& $1.356 \times 10^{-9} \mathrm{~m}(10,10)$ \\
Length $L$ & $(2.83-28.3) \times 10^{-9} \mathrm{~m}$ \\
\hline
\end{tabular}

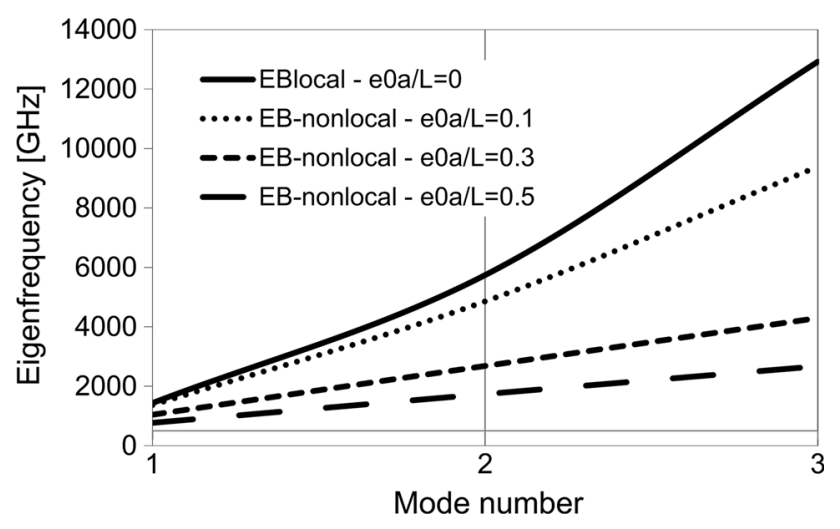

Fig. 2 Comparison of eigenfrequencies for the local and the nonlocal Euler-Bernoulli beam model for various $e_{0} a / L$ values for $(5,5) \mathrm{S}-\mathrm{S}$ nanobeam for $a=0.142 \mathrm{~nm}, L=2.83 \mathrm{~nm}$ (ratio a/ $\mathrm{L}=0.05)$ and $e_{0}=0,2,6,10$ 


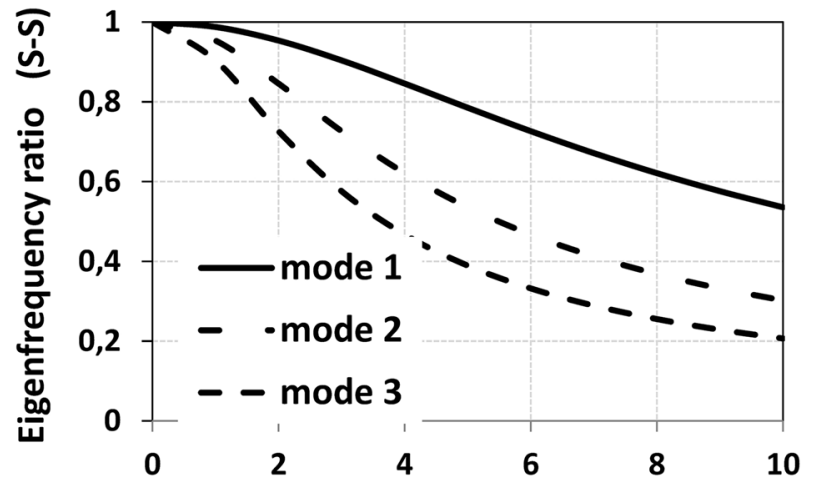

(a)
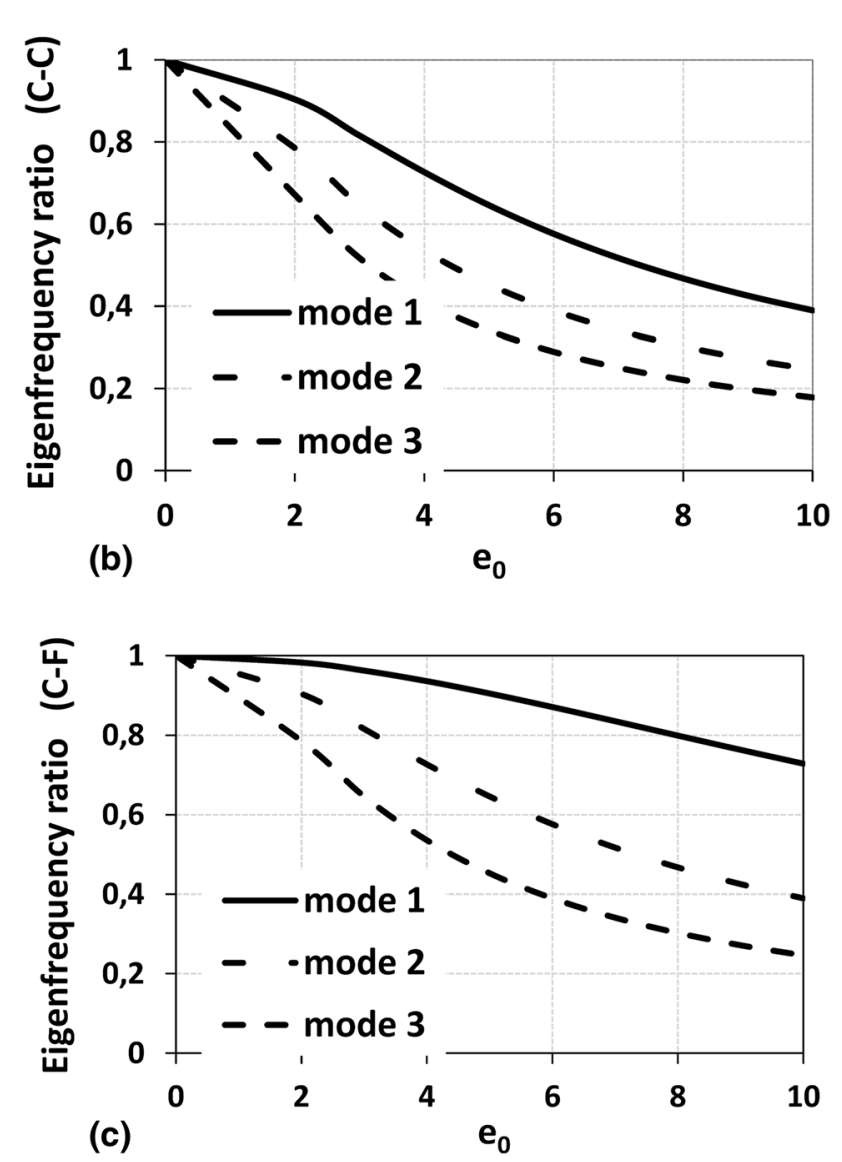

Fig. 3 Eigenfrequency ratio vs. nonlocal parameter for the first three modes: (a) S-S, (b) C-C and (c) C-F for $(5,5)$ SWCNT, $a=0.142 \mathrm{~nm}, L=2.83 \mathrm{~nm}($ ratio a $/ L=0.05$ )

the assumed nonlocal parameter, the lowest resonant eigenfrequencies are for $\mathrm{C}-\mathrm{C}$ nanotube, whereas the highest are for $\mathrm{C}-\mathrm{F}$ nanotube. The longer the nanotube is the lower influence of boundary conditions is observed.

\section{Numerical Verification}

To investigate the dynamic behavior of carbon nanotubes, a 3-D structural model based on FEM was built. Here, the carbon nanotube is presented as a space network of finite beam elements having circular cross section, as shown in Fig. 7.
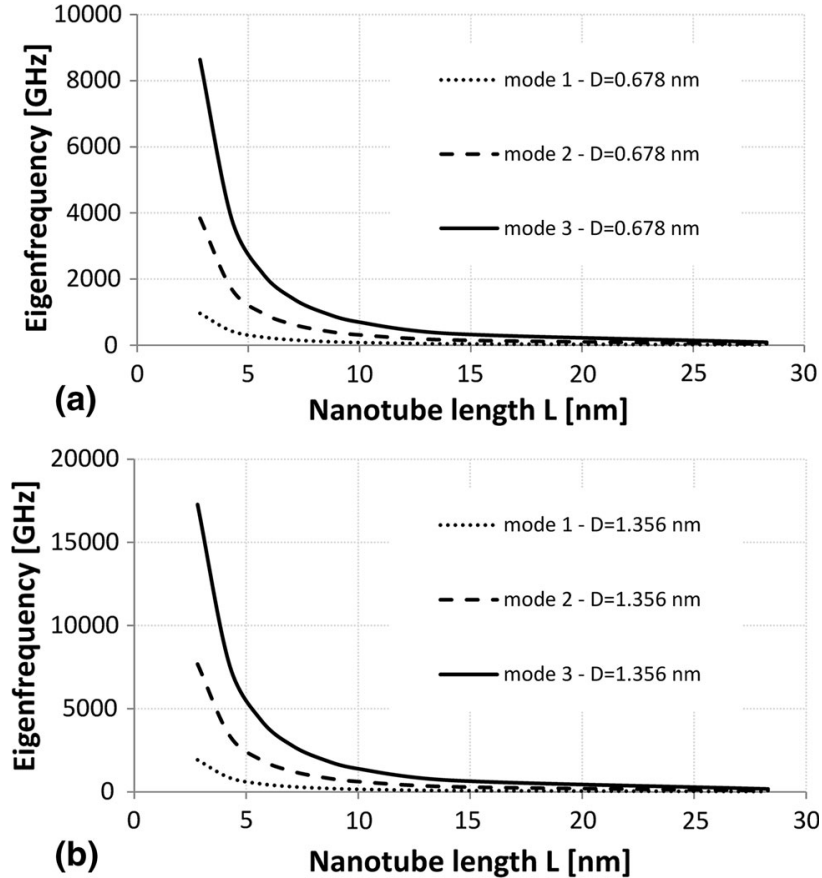

Fig. 4 Eigenfrequencies from local Euler-Bernoulli beam model vs. nanotube length $L$ for the first three modes: (a) $(5,5)$ nanotube-diameter $D=0.678 \mathrm{~nm}$, (b) $(10,10)$ nanotube-diameter $D=1.356 \mathrm{~nm}$ (S-S nanobeam case is presented; material data from Table 2)

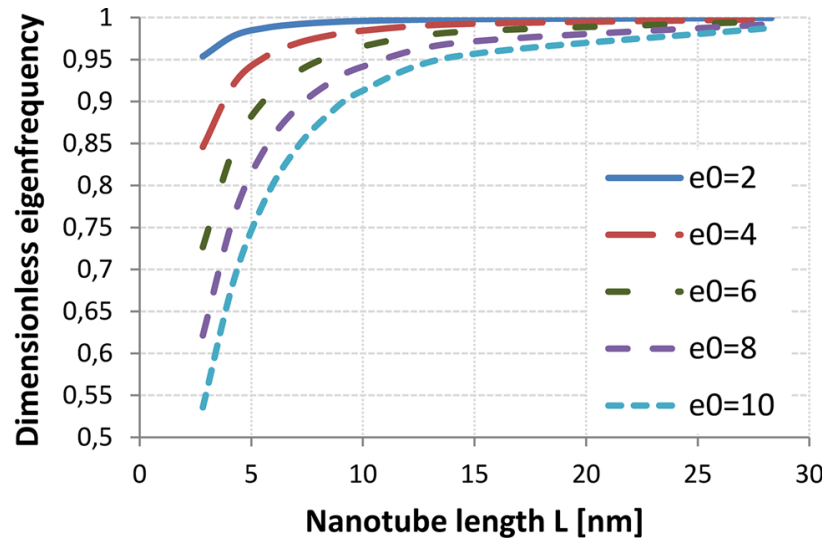

Fig. 5 Variation of dimensionless resonant eigenfrequency vs. nanotube length $L$ for various nonlocal parameters $e_{0}((5,5) \mathrm{S}-\mathrm{S}$ nanobeam case is presented; $a=0.142 \mathrm{~nm}$, material data from Table 2)

The commonly used approach to compute the elastic moduli of beam elements is a linkage between molecular and continuum mechanics. The linkage was initially proposed by Odegard et al. (Ref 63) and Li and Chou (Ref 64) and is used for example by (Ref 65-67). According to the molecularstructural mechanics approach, a SWCNT is presented as a space frame, in which the covalent $\mathrm{C}-\mathrm{C}$ bonds are represented as linking beams and the carbon atoms as joint nodes. Taking into account the energy equivalence between local potentials energies in computational chemistry and elemental strain energies in structural mechanics, the tensile resistance, the flexural rigidity, and the torsional stiffness for an equivalent 


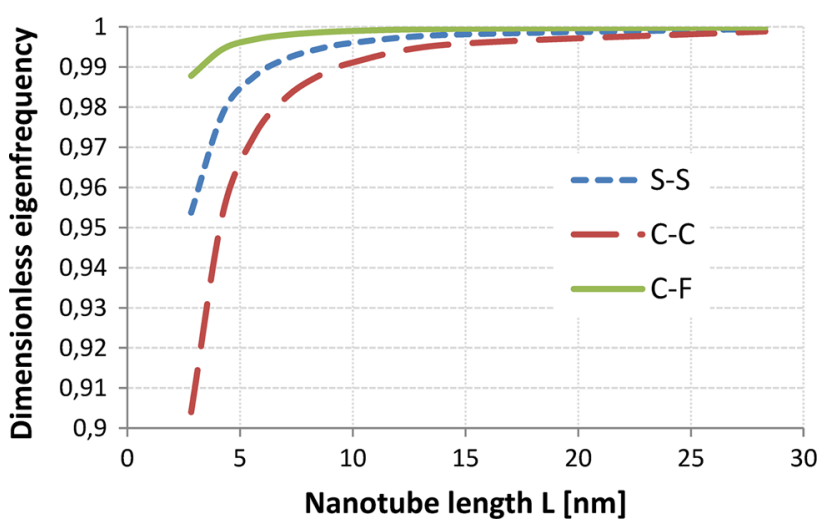

Fig. 6 Variation of dimensionless resonant eigenfrequency vs. nanotube length $L$ for various boundary conditions conducted for $(5,5)$ nanotube assuming nonlocal parameter $e_{0}=2, a=0.142 \mathrm{~nm}$ and $D=0.678 \mathrm{~nm}$

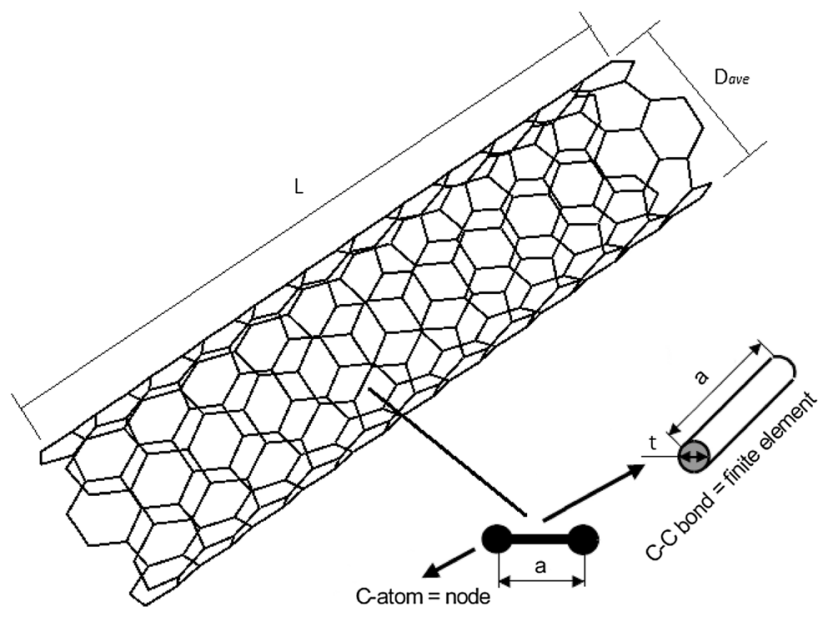

Fig. 7 Structural numerical model of $(5,5)$ SWCNT for $D_{\text {ave }}=0.674 \mathrm{~nm}, L=2.83 \mathrm{~nm}, a=0.142 \mathrm{~nm}, t=0.34 \mathrm{~nm}$

beam are computed (Ref $64,66,67)$. Mostly, the values of force field constants are based on the values for benzene, i.e., $k_{s}=652 \mathrm{~N} / \mathrm{m}, k_{\theta}=0.876 \mathrm{Nm} / \mathrm{rad}^{2}$, and $k_{\tau}=0.278 \mathrm{Nm} / \mathrm{rad}^{2}$ (Ref 63, 64). Based on the above force field constants and assuming the $\mathrm{C}-\mathrm{C}$ bond length $0.142 \mathrm{~nm}$ the cross-sectional diameter, moduli of elasticity and shear of the beam element are, respectively, $0.147 \mathrm{~nm}, 5.49 \mathrm{TPa}$ and 0.871 TPa (Ref 66). However, in the existing references, there are many force field constants applied in analyses of SWCNTs leading to different macroscopic material properties of nanostructure. The diverse values of the force field constants and the related elastic constants for beam elements used in FEM modeling are discussed by $\mathrm{Hu}$ et al. (Ref 68). The range of elastic properties for beam elements $\left(E_{b}\right.$ - beam Young's modulus and Poisson's ratio, $v_{b}$ ) representing the $\mathrm{C}-\mathrm{C}$ bonds according to analysis of force field constants is presented by Hu et al. (Ref 68) and is as follows: $E_{b}=(0.83-1.25) \mathrm{TPa}$ and $v_{b}=0.16-0.29$ assuming $\mathrm{C}-\mathrm{C}$ bond diameter $t=0.34 \mathrm{~nm}$. Also, in the paper by Domínguez-Rodríguez et al. (Ref 67), the sensitivity of FEA predictions to the bond force field constants is discussed taking as baseline the set of the bond force constants for benzene used, e.g., by (Ref 63). Besides the various force field constants applied in the modeling of carbon nanotubes, the scatter in the thicknesses of SWCNTs is addressed in the literature (Ref 6368). The wall thickness is presented in the range of 0.066 $0.34 \mathrm{~nm}$ (Ref 3), and commonly adopted value in FEA is $0.34 \mathrm{~nm}$ (Ref 68). Assuming the diameter of $\mathrm{C}-\mathrm{C}$ bond equal to the wall thickness of SWCNT $t=0.34 \mathrm{~nm}$, various bond stiffness of $\mathrm{C}-\mathrm{C}$ bond is applied in FEA, i.e., $E_{\mathrm{b}}=1.3 \mathrm{TPa}$ by Wernik and Meguid (Ref 69), $E_{\mathrm{b}}=1.16 \mathrm{TPa}$ by Tserpes et al. (Ref 70), $E_{\mathrm{b}}=1.06 \mathrm{TPa}$ by $\mathrm{Hu}$ et al. (Ref 68), $E_{\mathrm{b}}=1.04 \mathrm{TPa}$ by Shokrieh and Rafiee (Ref 66), $E_{\mathrm{b}}=1 \mathrm{TPa}$ Tongoyette et al. (Ref 71).

In the present structural modeling of SWCNT based on the literature review (Ref 3, 8, 63-71) and the earlier works of the author, e.g. (Ref 72), it was assumed for the single beam element representing the $\mathrm{C}-\mathrm{C}$ bond: the length $a=0.142 \mathrm{~nm}$, the stiffness $E_{\mathrm{b}}=1000 \mathrm{GPa}$, Poisson's ratio $v_{\mathrm{b}}=0.3$ and the bond diameter equal to the assumed nanotube thickness $t=0.34 \mathrm{~nm}$. For the beam element having circular cross section, the assumption here $E_{\mathrm{b}}=1000 \mathrm{GPa}$ and $t=0.34 \mathrm{~nm}$ gives the elastic constant $k_{s}=639 \mathrm{~N} / \mathrm{m}$ (see Ref 72), which is close to the value $k_{s}=652 \mathrm{~N} / \mathrm{m}$, e.g., in the work (Ref 63). To verify the present results of eigenfrequencies for assumed $E_{\mathrm{b}}$, $v_{\mathrm{b}}$, the elastic constants for the beam element were computed based on the force field constants used by Odegard et al. (Ref 63) and direct relationships between the structural mechanics parameters and the molecular mechanics parameters presented by Tserpes and Papanikos (Ref 65) for $t=0.34 \mathrm{~nm}$ and $a=0.142 \mathrm{~nm}$. The computed values were as follows: $E_{\mathrm{b}}=$ $1020 \mathrm{GPa}$ and $v_{\mathrm{b}}=0.16$. Next, the sensitivity of FEA predictions to the elastic constants of beam elements was analyzed. The values of eigenfrequencies for $E_{\mathrm{b}}=1020 \mathrm{GPa}$ and $v_{\mathrm{b}}=$ 0.16 were about $1-1.5 \%$ higher than for $E_{\mathrm{b}}=1000 \mathrm{GPa}$ and $v_{\mathrm{b}}=0.3$ used in the present numerical model. So, the results for $E_{\mathrm{b}}=1000 \mathrm{GPa}$ and $v_{\mathrm{b}}=0.3$ for the structural FE model of the nanotube are presented and discussed here.

In the current numerical modeling, the single-walled carbon nanotube $(5,5)$ with the average diameter $D_{\text {ave }}=0.674 \mathrm{~nm}$, the length $L=2.83 \mathrm{~nm}(L / D$ ratio $=4.2)$ having 240 carbon atoms was considered. Only the interatomic interactions between the closest neighbors in the $\mathrm{C}-\mathrm{C}$ network were considered (Ref 9, 63-65). The eigenfrequencies for the SWCNT for different boundary conditions resulted from numerical analyses are listed in Table 3. It is visible that the eigenfrequencies from FEM model are also very sensitive to the boundary conditions. The influence of BC is particularly visible for low modes, whereas for higher modes the differences become less visible.

To verify the present results from FEM modeling, a comparison of fundamental eigenfrequencies of SWCNTs with literature is listed in Table 4. Results shown in Table 4 are limited to the fundamental eigenfrequencies connected with short armchair SWCNTs having a small diameter. For small length, the influence of boundary conditions is highly visible (see Fig. 6 and Ref 9, 16, 31). The data in Table 4 are divided according to the length of $(5,5)$ nanotubes. The current results from the molecular-structural (MSM) FE model are compared with eigenfrequencies obtained from molecular dynamics simulations. Based on available data in the literature, the listed results show only values of fundamental eigenfrequencies for $\mathrm{C}-\mathrm{F}$ and $\mathrm{C}-\mathrm{C}$ boundary conditions however in the present work also S-S boundary conditions were analyzed. It is visible that the eigenfrequencies obtained in the current FE model are comparable with results from $\mathrm{MD}$ analyses presented by $\mathrm{Hu}$ et al. (Ref 31), Ansari et al. (Ref 13) and Zhang et al. (Ref 73) 
Table 3 FEM results of eigenfrequencies of $(5,5)$ SWCNT (GHz)

\begin{tabular}{lcccccrr}
\hline & \multicolumn{3}{c}{ Boundary conditions } & & \multicolumn{2}{c}{ Boundary conditions } \\
\cline { 2 - 4 } Mode no. & S-S & C-F & C-C & Mode no. & S-S & C-F \\
\hline 1 & 1383 & 373 & 1814 & 6 & 3544 & 2001 \\
2 & 1385 & 373 & 1817 & 7 & 5873 & 4079 \\
3 & 2001 & 1645 & 3370 & 8 & 5900 & 4084 \\
5 & 3321 & 1816 & 3932 & 9 & 5948 & 4946 \\
5 & 3540 & 1818 & 3937 & 10 & 5991 & 5665 \\
\hline
\end{tabular}

Table 4 Comparison of fundamental eigenfrequencies of SWCNTs with $\mathrm{C}-\mathrm{F}$ and $\mathrm{C}-\mathrm{C}$ boundary conditions with the literature

\begin{tabular}{|c|c|c|c|c|c|c|}
\hline \multirow[b]{2}{*}{$L(\mathbf{n m})$} & \multirow[b]{2}{*}{$L / D$} & \multirow[b]{2}{*}{ Chirality } & \multicolumn{2}{|c|}{ Fundamental eigenfrequency (GHz) } & \multirow[b]{2}{*}{ Method } & \multirow[b]{2}{*}{ Investigator(s) } \\
\hline & & & $\mathbf{C}-\mathbf{F}$ & $\mathrm{C}-\mathrm{C}$ & & \\
\hline 1.70 & 2.52 & $(5,5)$ & 550 & $\ldots$ & MD & Hu et al. (Ref 31$)$ \\
\hline 2.83 & 4.20 & $(5,5)$ & 373 & 1814 & MSM and FE & Present study \\
\hline 3.14 & 4.67 & $(5,5)$ & 247 & $\ldots$ & MD & Ansari et al. (Ref 13) \\
\hline 3.27 & 4.86 & $(5,5)$ & $\ldots$ & 1068 & MD & Zhang et al. (Ref 73) \\
\hline 4.12 & 6.12 & $(5,5)$ & 136 & 660 & MD & Hu et al. $(\operatorname{Ref} 31)$ \\
\hline 4.88 & 6.00 & $(6,6)$ & 150 & 710 & MSM & $\mathrm{Li}$ and Chou (Ref 16) \\
\hline
\end{tabular}

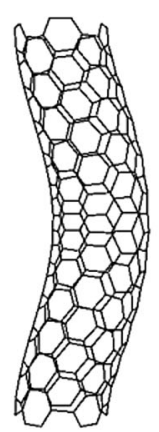

(a)

1

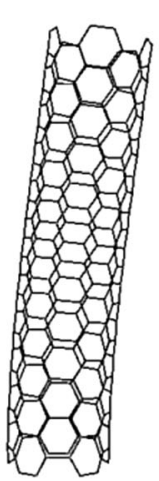

(b)

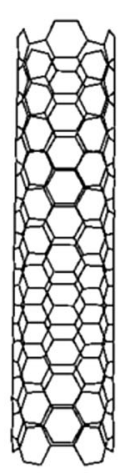

2

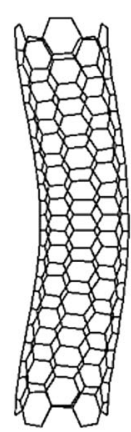

2

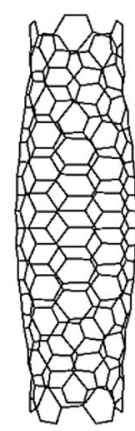

3

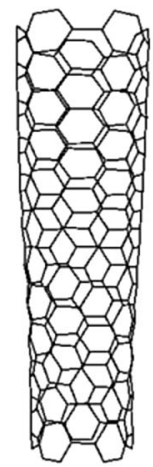

3

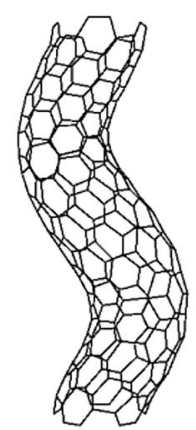

4

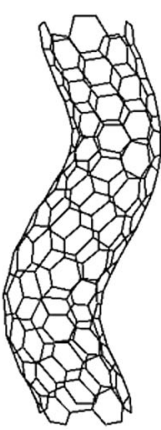

5

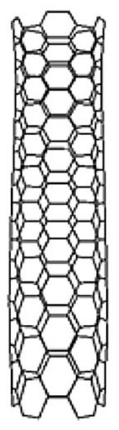

6

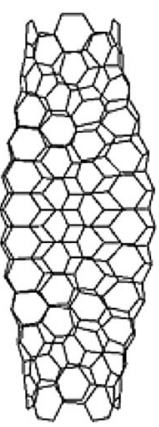

7

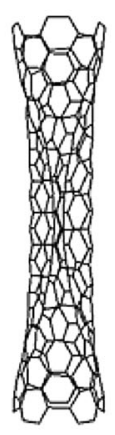

8

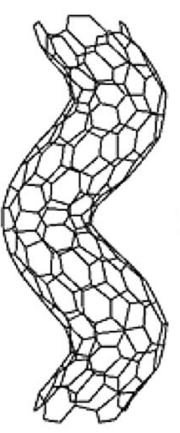

9

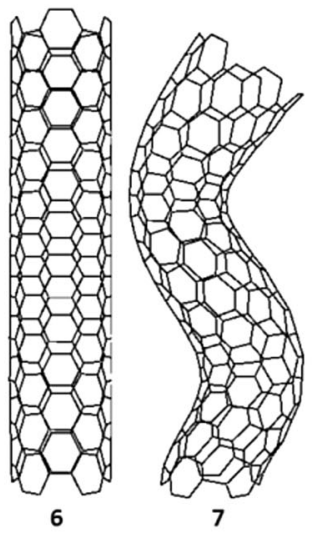

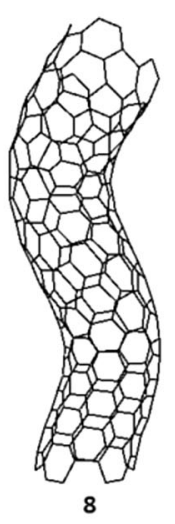
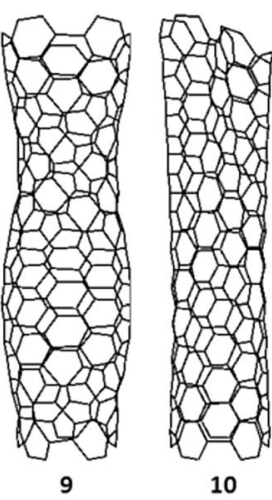

10

Fig. 8 Ten mode shapes of $(5,5)$ SWCNT with (a) C-C and (b) C-F boundary conditions

for short $(5,5)$ SWCNTs. The present results maintain the tendency of the decrease in eigenfrequencies in the function of increasing length of nanotube. In Table 4, the values obtained from MSM analysis are also presented for $(6,6)$ SWCNTs to demonstrate the influence of the diameter increase in nanotubes. For the very close value of $L / D$ being 6.12 for $(5,5)$ and 

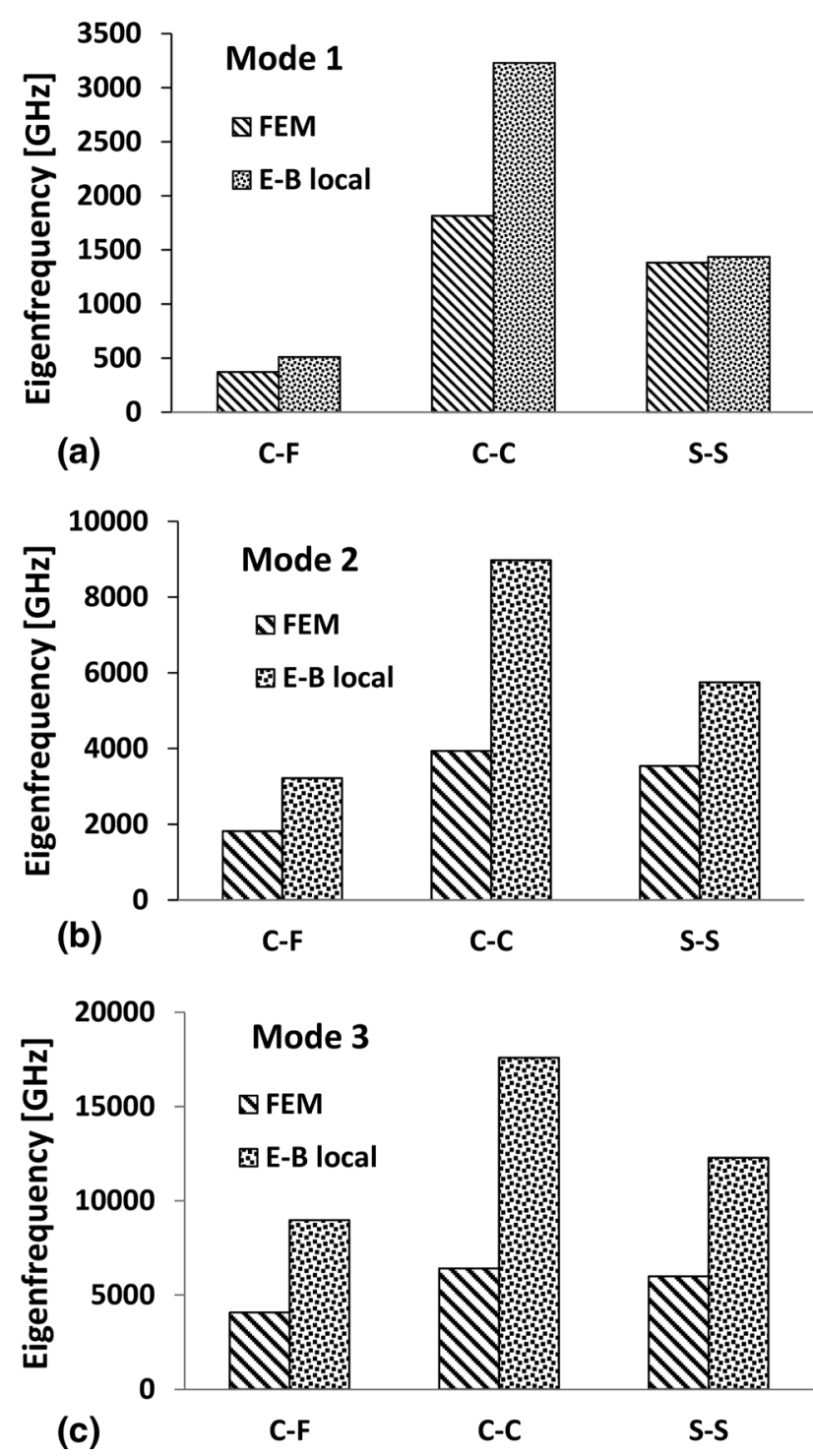

Fig. 9 The eigenfrequencies from the FEM analysis and the local Euler-Bernoulli model for (a) the first, (b) the second and (c) the third mode
6.00 for $(6,6)$ the higher frequencies are presented for the nanotube with higher diameter.

The numerical model based on the structural mechanic's approach allows presentation of all possible modes, including bending modes, axial modes, and radial breathing modes. The modes for $\mathrm{C}-\mathrm{C}$ and $\mathrm{C}-\mathrm{F}$ beam are presented in Fig. 8 .

\section{Nonlocal Parameter Fitting}

The FEM and analytical results of the eigenfrequencies have been contrasted to determine the nonlocal material parameter for the SWCNTs. The graphical comparison of CNT eigenfrequencies for first three modes resulted from the numerical structural FEM model, and the analytical Euler-Bernoulli model is presented in Fig. 9. It should be pointed out that the comparison takes into account only the flexural modes. Thus, Fig. 9 presents only, for example, for S-S boundary conditions the first, the sixth and the tenth mode resulted from the FEM analysis (see Table 3). The local beam model overpredicts the eigenfrequencies for the SWCNTs. It is especially noticeable for higher modes. For example, for the second mode for $\mathrm{C}-\mathrm{F}$ boundary conditions, the difference between the FEM results and the local beam model is about $80 \%$. In general, the values generated from the FEM are much lower than the values from the analytical local beam models. The difference in the eigenfrequencies varies versus boundary conditions.

In the present work, the selection of the most suitable smallscale material parameter $e_{0}$ for SWCNTs was based on the fitting the eigenfrequencies obtained from the analytical calculations to the numerical FEM results. The determination of the nonlocal parameter $e_{0}$ is presented in Table 5. The fitted value of the nonlocal parameter is not constant but depends on the boundary conditions. The smallest value of $e_{0}=2$ was determined for the simply supported boundary conditions, whereas the highest value of $e_{0}=10$ was computed for the cantilevered carbon nanotube. The nonlocal parameter determined for the cantilevered carbon nanotube is very close to the value $e_{0}=10.5$ presented in a paper of $\mathrm{Hu}$ et al. (Ref 31$)$ by fitting the local beam model to the molecular dynamics results for short CNTs. The calculations in Table 5 also revealed the

Table 5 The determination of the nonlocal small-scale parameter $e_{0}$ of $(5,5) \mathrm{SWCNT}$

\begin{tabular}{|c|c|c|c|c|c|}
\hline Mode no. & FEM & E-B local & $e_{0}$ & E-B nonlocal & Error, \% \\
\hline \multicolumn{6}{|c|}{ Eigenfrequency (GHz), S-S } \\
\hline 1 & 1383 & 1435 & 2 & 1368 & 1 \\
\hline 2 & 3544 & 5741 & 2 & 4856 & 37 \\
\hline 3 & 5991 & 12920 & 2 & 9384 & 56 \\
\hline \multicolumn{6}{|c|}{ Eigenfrequency $(\mathrm{GHz}), \mathrm{C}-\mathrm{C}$} \\
\hline 1 & 1813 & 3229 & 6 & 1860 & 2 \\
\hline 2 & 3931 & 8970 & 6 & 3494 & 11 \\
\hline 3 & 6411 & 17580 & 6 & 5084 & 20 \\
\hline \multicolumn{6}{|c|}{ Eigenfrequency $(\mathrm{GHz}), \mathrm{C}-\mathrm{F}$} \\
\hline 1 & 372 & 511 & 10 & 372 & 0 \\
\hline 2 & 1818 & 3229 & 10 & 1257 & 30 \\
\hline 3 & 4080 & 8970 & 10 & 2206 & 46 \\
\hline
\end{tabular}


increase in fitting errors for higher modes for all boundary conditions. So, not only the boundary conditions but also the mode number influence on the value of the nonlocal parameter. However, in the computations listed in Table 5, it was assumed that the resonant frequency is the most important for the CNTbased NEMS applications. Thus, the main point was to determine the nonlocal scale parameter for the first mode.

\section{Summary}

In the present work, the eigenfrequency comparison obtained from the finite element analysis and the analytical beam model was used to determine the magnitude of the nonlocal material parameter for the carbon nanotubes. In contrast to other works, the determination of eigenfrequencies involved FEM modeling instead of commonly used MD simulations. It was shown that the application of the nonlocal beam models involves analysis of the small-scale parameter, in which value determines the final eigenfrequencies and varies with both boundary conditions and the mode number. The proposed methodology involving the comparison between 3-D frame FEM results and local analytical beam models is helpful in the determination of nonlocal material parameter for SWCNTs. According to the present work, the following conclusions can be drawn:

- The highest values of eigenfrequencies are observed for the local beam model (Fig. 2).

- The eigenfrequencies increase with the mode number (Fig. 2).

- The vibration frequencies generally decrease with the increase in nonlocal parameter (Fig. 3).

- The vibration frequencies are mainly influenced by boundary conditions (Fig. 3).

- For long carbon nanotubes, the influence of nonlocal parameter is vanishing (Fig. 4 and 5).

- The increase in the diameter of SWCNT causes the increase in eigenfrequencies (Fig. 4).

- The influence of mode number on eigenfrequencies is visible for short nanotubes (Fig. 4).

- The influence of boundary conditions is vanishing for long SWCNTs (Fig. 5).

- In general, the values generated from the FEM analysis are much lower than the values from the analytical local beam models (Fig. 9).

- The numerical validation of nonlocal parameter revealed the high dependence on boundary conditions and mode number (Table 5).

The results presented in the paper are helpful in the investigation and design of carbon nanotubes-based NEMS in which nonlocal effects are significant.

\section{Open Access}

This article is distributed under the terms of the Creative Commons Attribution 4.0 International License (http://creativeco mmons.org/licenses/by/4.0/), which permits unrestricted use, distribution, and reproduction in any medium, provided you give appropriate credit to the original author(s) and the source, provide a link to the Creative Commons license, and indicate if changes were made.

\section{References}

1. S. Iijima, Helical Microtubules of Graphitic Carbon, Nature, 1991, 354, p $56-58$

2. S. Iijima and T. Ichihashi, Single-Shell Carbon Nanotubes of 1-nm Diameter, Nature, 1993, 363, p 603-605

3. O. Paris, Ed., Structure and Multiscale Mechanics of Carbon Nanomaterials, Springer, Berlin, 2016

4. H.B. Peng, C.W. Chang, S. Aloni, T.D. Yuzvinsky, and A. Zettl, Ultrahigh Frequency Nanotube Resonators, Phys. Rev. Lett., 2006, 97, p 087203

5. L. Ren, C.L. Pint, L.G. Booshehri, W.D. Rice, X. Wang, D.J. Hilton, K. Takeya, I. Kawayama, M. Tonouchi, R.H. Hauge, and J. Kono, Carbon Nanotube Terahertz Polarizer, Nano Lett., 2009, 9, p 2610-2613

6. G.W. Deng, D. Zhu, X.H. Wang et al., Strongly Coupled Nanotube Electromechanical Resonators, Nano Lett., 2016, 16, p 5456-5462

7. Muc A., Chwal M., Vibration Control of Defects in Carbon Nanotubes, in 2011, Proc. Symposium on Dynamics Modeling and Interaction Control in Virtual and Real Environments, Solid Mechanics and its Applications, vol. 30, pp. 239-246 (2011)

8. R.F. Gibson, E.O. Ayorinde, and Y.F. Wen, Vibrations of Carbon Nanotubes and Their Composites: A Review, Compos. Sci. Technol., 2007, 67, p 1-28

9. M. Chwał, Free Vibrations Analysis of Carbon Nanotubes, Adv. Mater. Res., 2014, 849, p 94-99

10. Poonia A., Dabas A., Chaudhary G., Kumar H., Singh K.K., Aggarwal L., Kumar A., Analysis of CNT Based Nano Bio-sensor for Virus Detection, in Proc. 14th IEEE Int. Conf. on Nanotechnology Toronto, Canada, August 18-21, pp. 747-751 (2014)

11. S. Badilescu and M. Packirisamy, BioMEMS: Science and Engineering Perspectives, CRC Press, Boca Raton, 2011

12. V.K. Khannan, Nanosensors: Physical, Chemical and Biological, CRC Press, Boca Raton, 2012

13. R. Ansari, S. Ajori, and B. Arash, Vibrations of Single- and DoubleWalled Carbon Nanotubes with Layerwise Boundary Conditions: A Molecular Dynamics Study, Curr. Appl. Phys., 2012, 12, p 707-711

14. J. Yoon, C.Q. Ru, and A. Mioduchowski, Timoshenko-Beam Effects on Transverse Wave Propagation in Carbon Nanotubes, Compos. B, 2004, 35, p 87-93

15. C.Y. Wang, C.Q. Ru, and A. Mioduchowski, Free Vibration of Multiwall Carbon Nanotubes, J. Appl. Phys., 2005, 97, p 114323

16. C. Li and T.W. Chou, Single-Walled Carbon Nanotubes as Ultrahigh Frequency Nanomechanical Resonators, Phys. Rev. B, 2003, 68, p 073405

17. D. Ambrosini and F. Borbon, On the Influence of the Shear Deformation and Boundary Conditions on the Transverse Vibration of Multi-walled Carbon Nanotubes, Comput. Mater. Sci., 2012, 53, p 214-219

18. Lee U., Oh H., You S., Natural Frequencies of Single-Walled Carbon Nanotubes, in Proc. 2nd IEEE Int. Conf. on Nano/Micro Engineered and Molecular Systems, Bangkok, Thailand, pp. 13-16 (2007)

19. A. Sakhaee-Pour, M.T. Ahmadian, and A. Vafai, Vibrational Analysis Of Single-Walled Carbon Nanotubes Using Beam Element, ThinWalled Struct., 2009, 47, p 646-652

20. V. Parvaneh, M. Shariati, and H. Torabi, Frequency Analysis of Perfect and Defective SWCNTs, Comput. Mater. Sci., 2011, 50, p 2051-2056

21. C.W. Fan, Y.Y. Liu, and C. Hwu, Finite Element Simulation for Estimating the Mechanical Properties of Multi-walled Carbon Nanotubes, Appl. Phys. A, 2009, 95, p 819-831

22. M. Rahmandoust and A. Öchsner, Buckling Behaviour and Natural Frequency of Zigzag and Armchair Single-Walled Carbon Nanotubes, J. Nano Res., 2011, 16, p 153-160

23. A. Ghavamian and A. Öchsner, Numerical Modeling of Eigenmodes and Eigenfrequencies of Single- and Multi-Walled Carbon Nanotubes Under the Influence of Atomic Defects, Comput. Mater. Sci., 2013, 72, p 42-48

24. J. Peddieson, G.R. Buchanan, and R.P. McNitt, Application of Nonlocal Continuum Models to Nanotechnology, Int. J. Eng. Sci., 2003, 41, p 305-312 
25. N. Anderson, A. Hartschuh, S. Cronin, and L. Novotny, Nanoscale Vibrational Analysis of Single-Walled Carbon Nanotubes, J. Am. Chem. Soc., 2005, 127, p 2533-2537

26. J.N. Reddy, Nonlocal Theories for Bending, Buckling and Vibration of Beams, Int. J. Eng. Sci., 2007, 45, p 288-307

27. Q. Wang, Q.K. Han, and B.C. Wen, Estimate of Material Property of Carbon Nanotubes Via Nonlocal Elasticity, Adv. Theor. Appl. Mech., 2008, 1, p 1-10

28. H. Heireche, A. Tounsi, A. Benzair, M. Maachou, and E.A. Adda Bedia, Sound Wave Propagation in Single-Walled Carbon Nanotubes Using Nonlocal Elasticity, Phys. E, 2008, 40, p 2791-2799

29. O. Civalek and C. Demir, Bending Analysis of Microtubules Using Nonlocal Euler-Bernoulli Beam Theory, Appl. Math. Model., 2011, 35, p 2053-2067

30. S. Narendar and S. Gopalakrishnan, Nonlocal Continuum Mechanics Formulation for Axial, Flexural, Shear and Contraction Coupled Wave Propagation in Single Walled Carbon Nanotubes, Latin Am. J. Sol. Struct., 2012, 9, p 497-513

31. Y.G. Hu, K.M. Liew, and Q. Wang, Nonlocal Continuum Model and Molecular Dynamics for Free Vibration of Single-Walled Carbon Nanotubes, J. Nanosci. Nanotechnol., 2011, 11, p 10401-10407

32. A. Tylikowski, Instability of Thermally Induced Vibrations of Carbon Nanotubes Via Nonlocal Elasticity, J. Therm. Stress., 2012, 35, p 281-289

33. M. Gurses, B. Akgoz, and O. Civalek, Mathematical Modeling of Vibration Problem of Nano-Sized Annular Sector Plates Using the Nonlocal Continuum Theory Via Eight-Node Discrete Singular Convolution Transformation, Appl. Math. Comput., 2012, 219, p 3226-3240

34. O. Civalek and C. Demir, A Simple Mathematical Model of Microtubules Surrounded by an Elastic Matrix by Nonlocal Finite Element Method, Appl. Math. Comput., 2016, 289, p 335-352

35. K. Mercan and O. Civalek, DSC Method for Buckling Analysis of Boron Nitride Nanotube (BNNT) Surrounded by An Elastic Matrix, Compos. Struct., 2016, 143, p 300-309

36. E.C. Aifantis, Gradient Deformation Models at Nano, Micro, and Macro Scales, J. Eng. Mater. Technol., 1999, 121, p 189-202

37. N.A. Fleck and J.W. Hutchinson, A Reformulation of Strain Gradient Plasticity, J. Mech. Phys. Solids, 2001, 49, p 2245-2271

38. D.C.C. Lam, F. Yang, A.C.M. Chong, J. Wang, and P. Tong, Experiments and Theory in Strain Gradient Elasticity, J. Mech. Phys. Solids, 2003, 51, p 1477-1508

39. B. Akgoz and O. Civalek, Buckling Analysis of Linearly Tapered Micro-Columns Based on Strain Gradient Elasticity, Struct. Eng. Mech., 2013, 48, p 195-205

40. B. Akgoz and O. Civalek, Bending Analysis of FG Microbeams Resting on Winkler Elastic Foundation Via Strain Gradient Elasticity, Compos. Struct., 2015, 134, p 294-301

41. B. Akgoz and O. Civalek, Bending Analysis of Embedded Carbon Nanotubes Resting on an Elastic Foundation Using Strain Gradient Theory, Acta Astronaut., 2016, 119, p 1-12

42. R.A. Toupin, Theory of Elasticity with Couple Stresses, Arch. Ration. Mech. Anal., 1964, 17, p 85-112

43. W.J. Chen and X.P. Li, Size-Dependent Free Vibration Analysis of Composite Laminated Timoshenko Beam Based on New Modified Couple Stress Theory, Arch. Appl. Mech., 2013, 83, p 431-444

44. Q. Wang, Wave Propagation in Carbon Nanotubes Via Nonlocal Continuum Mechanics, J. Appl. Phys., 2005, 98, p 124301

45. Q. Wang and V.K. Varadan, Vibration of Carbon Nanotubes Studied Using Nonlocal Continuum Mechanics, Smart Mater. Struct., 2006, 15, p 659-666

46. P. Lu, H.P. Lee, C. Lu, and P.Q. Zhang, Application of Nonlocal Beam Models for Carbon Nanotubes, Int. J. Sol. Struct., 2007, 44, p 52895300

47. H. Heireche, A. Tounsi, A. Benzair, M. Maachou, and E.A. Adda Bedia, Sound Wave Propagation in Single-Walled Carbon Nanotubes Using Nonlocal Elasticity, Physica E, 2008, 40, p 2791-2799

48. B. Arash and Q. Wang, A Review on the Application of Nonlocal Elastic Models in Modeling of Carbon Nanotubes and Graphenes, Comput. Mater. Sci., 2012, 51, p 303-313

49. R. Barretta and F.M. de Sciarra, Analogies Between Nonlocal and Local Bernoulli-Euler Nanobeams, Arch. Appl. Mech., 2015, 85, p 89-99
50. Y.G. Hu, K.M. Liew, Q. Wang, X.Q. He, and B.I. Yakobson, Nonlocal Shell Model for Elastic Wave Propagation in Single- and DoubleWalled Carbon Nanotubes, J. Mech. Phys. Sol., 2008, 56, p 3475-3485

51. S.A. Fazelzadeh and E. Ghavanloo, Nonlocal Anisotropic Elastic Shell Model for Vibrations of Single-Walled Carbon Nanotubes with Arbitrary Chirality, Compos. Struct., 2012, 94, p 1016-1022

52. S.C. Pradhan, Nonlocal Finite Element Analysis and Small Scale Effects of CNTs with Timoshenko Beam Theory, Finite Elem. Anal. Des., 2012, 50, p 8-20

53. M. Di Paola, G. Failla, A. Pirrotta, A. Sofi, and M. Zingales, The Mechanically Based Non-local Elasticity: An Overview of Main Results and Future Challenges, Phil. Trans. R. Soc. A, 2013, 371, p 20120433

54. M.A. Eltaher, M.E. Khater, and S.A. Emam, A Review on Nonlocal Elastic Models for Bending, Buckling, Vibrations, and Wave Propagation of Nanoscale Beams, Appl. Math. Model., 2016, 40, p 41094128

55. J. Fernandez-Saez, R. Zaera, J.A. Loya, and J.N. Reddy, Bending of Euler-Bernoulli Beams Using Eringen's Integral Formulation: A Paradox Resolved, Int. J. Eng. Sci., 2016, 99, p 107-116

56. C. Koutsoumaris, K.G. Eptaimeros, and G.J. Tsamasphyros, A Different Approach to Eringen's Nonlocal Integral Stress Model with Applications for Beams, Int. J. Solids Struct., 2017, 112, p 222-238

57. G. Romano, R. Barretta, M. Diaco, and F.M. de Sciarra, Constitutive Boundary Conditions and Paradoxes in Nonlocal Elastic Nanobeams, Int. J. Mech. Sci., 2017, 121, p 151-156

58. A.C. Eringen, Nonlocal Polar Elastic Continua, Int. J. Eng. Sci., 1972, 10, p $1-16$

59. A.C. Eringen and D.G.B. Edelen, On Nonlocal Elasticity, Int. J. Eng. Sci., 1972, 10, p 233-248

60. C.M. Wang, Y.Y. Zhang, and X.Q. He, Vibration of Nonlocal Timoshenko Beams, Nanotechnol., 2007, 18, p 105401

61. A.C. Eringen, On Differential Equations of Nonlocal Elasticity and Solutions of Screw Dislocation and Surface Waves, J. Appl. Phys., 1983, 54, p 4703-4710

62. A. Krishnan, E. Dujardin, T.W. Ebbesen, P.N. Yianilos, and M.M.J. Treacy, Young's Modulus of Single-Walled Nanotubes, Phys. Rev. B, 1998, 58, p 14013

63. G.M. Odegard, T. Gates, L.M. Nicholson, and K.E. Wise, EquivalentContinuum Modeling of Nano-Structured Materials, Compos. Sci. Technol., 2002, 62, p 1869-1880

64. C. Li and T.W. Chou, A Structural Mechanics Approach for the Analysis of Carbon Nanotubes, Int. J. Solids Struct., 2003, 40, p 24872499

65. K.I. Tserpes and P. Papanikos, Finite Element Modeling of SingleWalled Carbon Nanotubes, Compos. B, 2005, 36, p 468-477

66. M.M. Shokrieh and R. Rafiee, Prediction of Young's Modulus of Graphene Sheets and Carbon Nanotubes Using Nanoscale Continuum Mechanics Approach, Mater. Des., 2010, 31, p 790-795

67. G. Domínguez-Rodríguez, A. Tapia, and F. Avilés, An Assessment of Finite Element Analysis to Predict the Elastic Modulus and Poisson's Ratio of Singlewall Carbon Nanotubes, Comput. Mater. Sci., 2014, 82, p 257-263

68. N. Hu, H. Fukunaga, C. Lu, M. Kameyama, and B. Yan, Prediction of Elastic Properties of Carbon Nanotube Reinforced Composites, Proc. R. Soc. A, 2005, 461, p 1685-1710

69. J.M. Wernik and S.A. Meguid, Atomistic-Based Continuum Modeling of the Nonlinear Behavior of Carbon Nanotubes, Acta Mech., 2010, 212, p 167-179

70. K.I. Tserpes, P. Papanikos, and S.A. Tsirkas, A Progressive Fracture Model for Carbon Nanotubes, Compos. B, 2006, 37, p 662-669

71. C. Thongyothee, S. Chucheepsakul, and T. Li, Nonlocal Elasticity Theory for Free Vibration of Single-Walled Carbon Nanotubes, Adv. Mater. Res., 2013, 747, p 257-260

72. M. Chwał, Influence of Vacancy Defects on the Mechanical Behavior and Properties of Carbon Nanotubes, Procedia Eng., 2011, 10, p 1579_ 1584

73. Y.Y. Zhang, C.M. Wang, and V.B.C. Tan, Assessment of Timoshenko Beam Models for Vibrational Behavior of Single-Walled Carbon Nanotubes Using Molecular Dynamics, Adv. Appl. Math. Mech., 2009, 1, p 89-106 